\title{
Civilisations
}

Revue internationale d'anthropologie et de sciences

humaines

39 | 1991

Japon : les enjeux du futur

\section{Le système éducatif japonais en question}

\section{Jean-François Sabouret}

URL : http://journals.openedition.org/civilisations/1654

DOI : 10.4000/civilisations. 1654

ISSN : 2032-0442

\section{Éditeur}

Institut de sociologie de l'Université Libre de Bruxelles

\section{Édition imprimée}

Date de publication : 30 octobre 1991

Pagination : 129-159

ISBN : 2-87263-044-9

ISSN : 0009-8140

\section{Référence électronique}

Jean-François Sabouret, "Le système éducatif japonais en question », Civilisations [En ligne],

39 | 1991, mis en ligne le 06 juillet 2009, consulté le 19 avril 2019. URL : http://

journals.openedition.org/civilisations/1654; DOI : 10.4000/civilisations.1654 


\title{
LE SYSTEME EDUCATIF JAPONAIS EN QUESTION
}

\author{
Jean-François SABOURET
}

\section{Introduction}

L'éducation comme un droit de l'homme : l'idéal de l'aprèsguerre

Depuis de longues années déjà l'école est en Europe un lieu d'âpres affrontements entre divers courants, diverses tendances faisant ressortir des intérêts divergents voire opposés : qu'ils soient d'ordres politiques, parentaux, régionaux, sexuels, ethniques... ces voix qui parlent se font entendre pour défendre leur vision de l'école au nom de l'égalité ou du respect des capacités ou des dons des personnes. Que se passe-t-il ?

La commission Langevin-Wallon exprimait déjà dans les premières années de l'après-guerre (juin 1947), cette volonté des Français de donner à leurs enfants plus d'éducation qu'ils n'en avaient reçu eux-mêmes et ceci afin de réunir le maximum de chances de réussite dans leur vie adulte professionnelle.

En Angleterre et au Pays de Galles, l'acte d'éducation de 1944 , disait à peu près la même chose. En Allemagne, en Belgique, en Hollande aussi. Dans une Europe détruite par la guerre, et alors que les responsables devaient faire face à des problèmes très concrets de bâtiments scolaires à reconstruire, à aménager, d'enseignants à recruter... de nombreux pays européens jetèrent les bases de principes clairs défendant généreusement l'idée selon laquelle chaque individu doit pouvoir accéder à l'école et y développer ses aptitudes et capacités jusqu'au plein épanouissement de celles-ci et ce quelque soit son origine sociale. Le droit à l'éducation 
s'affirmait plus fortement dans les années d'après-guerre et l'on aurait pu penser qu'il allait être le moteur dont la résultante serait un brassage social plus grand. Dans les pays d'Europe, on affirmait partout les principes de "l'égalité devant l'éducation" et "l'éducation comme l'un des droits de l'homme".

Si l'on applique les mesures concrètes de ces idéaux cela se traduit par un nombre accru d'enfants à l'école primaire et en premier cycle, ensuite par une multiplication des filières, par le développement des spécialisations, par l'accès aux études supérieures d'un nombre d'étudiants toujours en augmentation et aussi, bien sûr, par le recrutement d'un grand nombre d'enseignants ayant une formation plus appropriée propre à répondre aux besoins d'une population à la fois plus nombreuse et plus hétérogène.

De plus l'idée générale qui sous-tendait ces projets était de réaliser ces principes dans le contexte plus vaste de la disparition d'une des barrières principales à l'égalité en matière scolaire : l'argent. Chaque pays européen fit donc de grands efforts pour réaliser le principe de la gratuité, et pour corriger par de nombreuses bourses les inégalités sociales dues aux manque de ressources financières des familles les plus modestes.

Malheureusement le projet Langevin-Wallon dans son ensemble fut rejeté car jugé trop coûteux; en Angleterre on manqua d'enseignants; en Allemagne de l'Ouest il fallait faire face au problème de la pression démographique, dû à la conséquence de la politique nataliste des Nazis au début des années quarante et à l'arrivée de millions de réfugiés.

En outre, on a assisté, dans les années cinquante, à une montée d'une résistance à une trop grande démocratisation au nom d'une tradition de la qualité académique de l'enseignement 
traditionnel au sein des lycées en France, des Gymnasium en Allemagne, des Grammar School en Grande-Bretagne.

Tous ces problèmes, toutes ces luttes pour défendre des positions acquises ou pour imposer des idéaux nouveaux ont dominé la vie de l'enseignement en Europe pendant une bonne trentaine d'années.

\section{Education et compétition}

Depuis le début des années quatre-vingts, on s'interroge beaucoup en Europe à nouveau sur la nécessité de promouvoir un enseignement plus adapté à l'évolution du monde moderne et de faire un sort aux savoirs inutiles. Les idéaux qui furent ceux de l'après-guerre ne sont pas récusés, certes, et la plupart révèrent comme il se doit l'idéal du plan Langevin-Wallon comme une table de la loi pédagogique qu'il serait bon de vivre mais beaucoup ajoutent que la réalité quotidienne est celle d'un environnement économique sévère et qu'il s'agit de favoriser les savoir faire des individus capables de "faire la différence" technologique, scientifique par rapport aux voisins et concurrents.

Les pays européens se débattent dans des problèmes et des solutions en matière d'éducation finalement assez proches, si on les compare à ceux, que peuvent rencontrer les sociétés industrialisées de cultures non-occidentales dont les prouesses économiques attirent l'attention de l'Occident et d'une certaine façon contribuent à ressérer ses liens, par la menace qu'il fait peser sur ses équilibres traditionnels.

Cependant cette menace est porteuse de curiosité. On a cherché à savoir pourquoi certains pays asiatiques avaient des taux de croissance plus importants que ceux des pays occidentaux. Parmi les réponses avancées on a bien sûr parlé du 
bas prix de la main-d'oeuvre, des faibles salaires des travailleurs, de la protection sociale insignifiante, des congés inexistants, et de l'indifférence aux personnes retraitées confiées à leurs descendants au nom d'une tradition millénaire. Il y a du vrai dans ces raisons mais elles ne suffisent plus à masquer un phénomène important sur lequel on ne s'est pas suffisamment penché en Europe jusqu'à ces dernières années et qui est celui de l'éducation : les pays asiatiques de culture confucéenne ont fait un formidable investissement en matière d'éducation en même temps qu'elles investissaient dans le développement économique et industriel de leurs pays respectifs comme si les deux étaient liés.

Alors qu'en Europe une politique industrielle reposant sur de bas salaires était alimentée par une main-d'oeuvre dépourvue d'éducation et de formation, main-d'oeuvre venue des colonies ou ex-colonies, ou des pays voisins plus pauvres, les pays asiatiques eux recrutaient, pour un travail égal, des personnes d'années en années toujours plus diplômées.

Pourquoi des bacheliers, disait-on il y a peu, pour assembler des voitures dans les usines automobiles ? Pourquoi étudier quatre années après le bac quand on est jeune fille pour devenir secrétaire assujettie à des tâches subalternes? Ces questions se posent de moins en moins en Europe car on est de plus en plus convaincu qu'il y a quelque chose à apprendre du Japon, promu au rang de modèle par certains. Le Japon a fait une entrée fracassante dans le monde industriel et est sans conteste la figure emblématique entre tous. On sait qu'il y a un lien causal entre le haut niveau d'éducation des Japonais dans leur ensemble et les performances réalisées par ce pays. C'est donc sur l'éducation japonaise, prise dans son ensemble, que se tournent de plus en plus de regards, que se concentrent des missions d'études ou des enquêtes approfondies. 
Depuis la fin de la guerre, les pays européens ont procédé à de nombreuses réformes éducatives. La France de ce côté là est exemplaire; chaque ministre de l'éducation ayant à coeur de faire autre chose que son prédécesseur. Depuis le début des années 80 , on considère davantage l'éducation sous l'angle d'une formation nécessaire pour permettre à un pays de mieux s'adapter à l'environnement économique, de mieux riposter à l'agressivité d'autres pays industrialisés au premier rang desquels se trouve le Japon. Le titre d'un des ouvrages de JeanPierre Chevénement, alors ministre de l'éducation est de ce point de vue là significatif : Apprendre pour entreprendre. Estce à dire que les responsables de l'éducation en France vont abandonné, d'une certaines façon, l'esprit d'égalité qui a présidé à la rédaction du plan Langevin-Wallon et auquel on s'est toujours beaucoup référé jusqu'à ces dernières années? Il apparaît donc indispensable maintenant d'examiner l'éducation japonaise et de voir si certains aspects de celle-ci peuvent être porteur d'enseignement pour nous européens et sont compatibles avec nos idéaux éducatifs.

L'éducation japonaise est très souvent accompagnée du qualificatif "performant" comme s'il s'agissait d'un secteur industriel. Pourtant elle est loin de ne rencontrer aucun obstacle et les Japonais s'étonnent souvent eux-mêmes quand ils entendent dire que leur école est un modèle pour certains pays étrangers:

Le système éducatif japonais est confronté à bien des problèmes qui ne sont pas toujours si éloignés que cela des problèmes éducatifs rencontrés en Europe. En particulier, il faut briser d'emblée le mythe du consensus, s'il en a jamais existé un, au sujet de l'école japonaise car celle-ci est un lieu de contestation où des projets de réforme divers voire opposés s'affrontent. Derrière ces projets, ce sont des idéaux différents de l'homme qui sont en présence. Les Japonais ne partagent pas 
tous le même idéal éducatif même si le parti conservateur qui domine toutes la vie politique du Japon d'après-guerre impose lourdement sa vision de l'homme japonais, son éthique, et le rôle qu'il doit jouer tant au sein de la société japonaise qu'à l'extérieur. 2. L'éducation au Japon est un enjeu politique et un lieu de haute
contestation

Tant à l'extérieur qu'à l'intérieur du Japon, les discours que l'on tient sur l'éducation japonaise sont contradictoires. Il en est de cette dernière comme de l'histoire bien connue d'un homme se trouvant assis devant une bouteille dont le contenu est entamé de moitié. Quel parti prendre ? Un pessimiste dira qu'elle est à moitié vide et un optimiste à moitié pleine.

De la même façon, il est possible de commencer par les aspects négatifs de l'école japonaise sur lesquels on a tant insisté à l'étranger déjà : les suicides d'écoliers, la violence à l'école, les phénomènes de souffre-douleur, l'enfer de la préparation des concours, le coût très élevé de la scolarité au lycée et à l'université, l'éducation vu comme un marché très commercial avec le développement des désormais célèbres juku et yobikô.

On peut également emboucher les trompettes gouvernementales pour annoncer, sur la musique de l'hymme à la joie, les taux records de lycéens sortant à dix-huit ans de leurs établissements avec un diplôme en poche. $92 \%$ d'une classe d'âge. Qui dit mieux ? On peut continuer en montrant que la tendance chez les Japonais à poursuivre des études au-delà de 18 ans s'affirme chaque année. Des spécialistes estiment qu'en l'an 2000, toute une classe d'âge ou presque étudiera quelque chose jusqu'à 20 ans au moins. 
A la même époque, la France, qui se débat dans de multiples problèmes de recrutement de professeurs, de salaires d'enseignants, d'infrastructures scolaires insuffisantes, promet de faire obtenir le baccalauréat à $80 \%$ d'une classe d'âge. Le temps passant et l'objectif apparaissant comme irréalisable, on commence dans les sphères des responsables au ministère de l'éducation à nuancer ces propos anciens en affirmant que les objectifs étaient non pas de faire obtenir le baccalauréat à une fraction aussi importante d'une classe d'âge mais de faire parvenir cette classe d'âge au niveau du baccalaurat et compte tenu des pourcentages d'échec à cet examen, on ne pourrait compter que sur $52 \%$ environ de bacheliers par classe d'âge. A quelles calendes grecques faut-il renvoyer les $80 \%$ de bacheliers en France. Quoiqu'il en soit, le taux de $80 \%$ de lycéens diplômés au Japon sera une performance vieille de 32 ans (1968) au début du troisième millénaire.

Un autre aspect important différencie le rythme de présence à l'école des pédagogues français et japonais : les enseignants français ne sont pas tenus de demeurer dans leurs établissements en dehors de leurs heures de cours mais au Japon ils doivent comme tous les fonctionnaires effectuer 40 heures minimum de présence dans leur établissement et ce qu'ils aient cours ou non.

Il y a de quoi faire rêver plus d'un ministre de l'éducation nationale européen. Sans l'excellence des divers systèmes éducatifs qui se sont succédé au Japon depuis la restauration de Meiji (1868) il serait difficile d'expliquer comment le Japon a non seulement rattrapé son retard mais a même, dans bien des domaines, déjà dépassé l'occident, le Japon ayant fait sien le slogan d'un des plus grands intellectuels et éducateur de cette période nipponne des lumières, Fukuzawa Yukichi (1834-1901), lorsqu'il proposait aux Japonais : "de rattraper et de dépasser l'occident". Sans une éducation adaptée aux besoins du pays à 
chaque moment de son histoire, il ne peut y avoir de réussite économique, scientifique et de progrès social pour un peuple.

Comment donc présenter dans sa vérité cette éducation japonaise tant prisée par les uns et décriée par les autres ? Que peut-on dire d'un système éducatif qui est tantôt un repoussoir tantôt un paragon selon les besoins de la plaidoirie ? Les arguments et les faits sont jetés à la face des uns et des autres comme s'ils s'agissait de programmes politiques dans un débat électoral car derrière ces chiffres et exemples, c'est sans doute toute une conception de l'homme qui se cache. Il faut donc chercher jusque-là pour essayer de comprendre ce que les hommes de pouvoir d'un côté et les éducateurs de l'autre attendent de l'éducation.

Cette sorte de machine à fabriquer les hommes qu'est un système éducatif doit-elle tourner pour le plus grand profit de l'état et les objectifs de ceux qui ont en main le pouvoir ou bien doit-elle faire en sorte que la personnalité et les talents de chaque individu puissent s'épanouir dans le plus grand respect des droits de l'enfant et du devenir de chacun?

\section{Un système éducatif datant de l'occupation américaine}

Le système éducatif que connaît le Japon actuel date des années 47, époque durant laquelle l'armée d'occupation américaine et le SCAP (Supreme Commander for the Allied Powers) travaillait à remodeler un nouveau visage, démocratique celui-là, au Japon vaincu, exsangue. Des missions éducatives, venues des Etats-Unis, cherchaient ainsi à extirper à jamais dans l'esprit de la jeunesse le mal qu'avait été le fanatisme de la cause impériale du grand Japon.

Le mal, c'était justement toute l'éducation de la période militariste, celle de l'obéissance aveugle devant les ordres des 
supérieurs, du don de soi à la personne sacrée de l'empereurdieu, Hiro-Hito, monté sur le trône en 1926. Tous les manuels scolaires de morale et de langue japonaise, entre autres des années qui ont précédé la guerre et jusqu'à la défaite sont pleins de ces discours de sacrifice et de cette morale édifiante.

Il était lu chaque jour aux enfants des écoles primaires et secondaires le rescrit impérial du 30 octobre 1890 (kyôiku chokugo), récit dans lequel il était enseigné que l'empereur était le père de la nation japonaise à qui chacun devait fidélité et sacrifice total en cas de besoin. La seconde guerre mondiale a montré à tous jusqu'où pouvait aller cette idéologie du don de soi et de l'obéissance aveugle.

Toutes les personnes qui ont fréquenté l'école primaire jusqu'au moment de la défaite, sont capables encore de réciter de nos jours cette table de la loi nipponne tant ils en furent imprégnés. La plupart des dirigeants conservateurs actuels ont eux aussi subi l'empreinte de cette éducation bien particulière et l'on peut se demander si, d'une certaine façon, les nostalgiques de l'ancien système éducatif qui ont non seulement des idées mais aussi du pouvoir quand ils sont membres du parti libéral démocrate, ne cherchent pas purement et simplement à restaurer cette ancienne morale qui, assénée à des générations entières, a apporté le malheur au Japon et dans toute l'Asie du sud-est.

Parce que la valeur de ce texte est non seulement historique mais aussi actuelle et parce que celui-ci guide l'esprit de bien des hommes politiques et des commissions de réforme de l'éducation qui gravitent autour du premier ministre, il apparait indispensable de le rappeler ici :

"Nos ancêtres impériaux ont fondé notre empire sur une base large et durable et ont profondément et fermement 
implanté la vertu; nos sujets, toujours unis dans la loyauté et la piété filiale en ont, de génération en génération, illustré la beauté. C'est la gloire du caractère fondamental de notre empire et là se trouve aussi la source de notre éducation. Vous, nos sujets, montrez-vous filiaux vis-à-vis de vos parents, affectionnés vis-à-vis de vos frères et soeurs; qu'entre mari et femme règne l'harmonie, entre amis la franchise; tenez-vous vous-mêmes dans la modestie et la modération; étendez à tous votre bienveillance; poursuivez l'étude et cultivez les arts et de cette façon, développez vos facultés intellectuelles et perfectionnez votre puissance morale; au-delà, faites progresser le bien public et favorisez les intérêts communs; respectez toujours la constitution et observez les lois; si jamais un danger se présente, offrez-vous courageusement à l'état; et ainsi gardez et maintenez la prospérité de notre trône impérial, aussi durable que le ciel et la terre. Ainsi, vous ne serez pas seulement nos sujets bons et loyaux, mais vous rendrez illustres les meilleures traditions de nos ancêtres.

La voie présentée ici est assurément l'enseignement dispensé par nos ancêtres impériaux : il doit être observé de la même façon par leurs descendants et les sujets car il est infaillible pour tous les âges et vrai en tous lieux. Notre voeu est de la mettre en commun avec vous, nos sujets, en toute révérence dans nos coeurs, afin que nous atteignions tous ainsi à la même vertu" (Yoshida K et Kaigo T., Japanese education, PP2-3 cité in la civilisation japonaise de Vadim et Danielle Elisseeff, pp. 214, 215, Ed. Les Grandes civilisations/Arthaud.

\section{Le bilan de l'après-guerre : une nouvelle éducation au goût ancien}

C'est dans un Japon sous l'autorité de l'ennemi d'hier que les simples soldats et les officiers doivent rentrer et apprendre la démocratie à l'américaine. Les personnalités politiques les plus marquantes sont emprisonnées, certaines jugées et même 
condamnées à la pendaison. Sur les 25 accusés du tribunal de Tôkyô (qui est en quelque sorte le pendant de celui de Nüremberg) sept sont pendus dont le général Tôjo Hideki et l'ex-premier ministre Hirota. D'autres criminels de guerre, suffisamment coupables pour avoir été classés par les Américains dans la catégorie A, sont emprisonnés et attendent un jugement. Mais la guerre froide vient bouleverser la politique des Américains vis-à-vis des coupables d'hier.

L'heure n'est plus au procès de l'idéologie passée mais à la lutte contre le mal absolu, la gangrène idéologique présente qui gagne l'extrême-orient. La grande Chine tombe totalement entre les mains de Mao-Tsé Toung et des communistes en 1949 (prise de Pékin le 22 janvier 1949). La guerre de Corée commence le 25 juin 1950. Au Japon même, les mouvements de gauche sont puissants. En échange de leur collaboration active contre le mal rouge que représente le communisme, les Américains libèrent de nombreux prisonniers politiques. Certains vont même prospérer et participer activement à la reconstruction économique du pays. Monsieur Kishi Nobusuke deviendra même premier ministre (février 57 à juillet 60 ). Deux anciens criminels de guerre de catégorie A, liés à l'extrêmedroite ainsi qu'à la pègre nipponne, Sasakawa Ryoichi et Kodama Yoshio vont jouer un rôle capital dans la réorganisation de la droite japonaise et le développement du parti actuellement au pouvoir depuis plus de 40 ans : le Jiminto (Parti libéral démocrate). De nombreux anciens soldats ou officiers comme Nakasone Yasuhiro, qui n'ont rien oublié, rien renié de leur idéal farouchement nationaliste, sont déjà élus députés au moment de l'occupation américaine.

Si l'on considère les discours tenus au fil des ans par ces personnes influentes de la droite, notamment vis-à-vis de l'éducation et les idées de réforme, on comprend d'une certaine façon où elles veulent en venir lorsqu'elles mettent sur pied, 
comme Monsieur Nakasone l'a fait, une commission de réforme de l'éducation. La démarche "éducative" de ces hommes politiques (qui ont reçu l'empreinte et le souvenir de l'ancienne éducation, celle du rescrit impérial) pour être totalement comprise doit être appréciée dans le contexte d'une "posture politique" plus large qui dépasse forcément l'éducation strictosensu.

Ce sont les mêmes hommes politiques qui décident de dépasser le $1 \%$ du budget global de l'état pour les dépenses militaires. Ce sont encore ces mêmes personnes qui veulent réformer la constitution et le système éducatif. Cette démarche d'ensemble est cohérente et préméditée et c'est de cela qu'au travers de l'étude des fonctions et dysfonctions du système éducatif japonais actuel qu'il va être question ici.

\section{Rappel historique du problème}

Si l'on fait exception de la brève période pendant laquelle le parti socialiste a été au pouvoir après la guerre (mai 47 à mars 48) la vie politique japonaise a été et est encore dominée par la droite. Qu'on en juge par les chiffres. Les élections législatives de juillet 86 ont donné 304 sièges au parti libéral-démocrate sur un total de 512. L'alternance au pouvoir est un mot depuis longtemps réservé aux démocraties étrangères. Pourquoi, semblent dire les Japonais en votant massivement pour le PLD (LDP en anglais), congédierait-on une équipe qui gagne ? Le Japon est un pays riche et puissant. Dans les années 68, son PNB était le troisième de la planète. Aujourd'hui, il est le second. Et l'importance qu'il prend aux Etats-Unis même n'est pas sans en inquiéter plus d'un.

Cette excellence économique dont la rationalisation remonte aux années 1965, à l'époque dite de la haute croissance, a cependant son revers sur le plan humain. Ce n'est 
pas seulement le lieu de travail, mais aussi la vie familiale, l'école et les lieux de sociabilité traditionnels qui ont été profondément altérés voire sacrifiés sur l'autel de l'économisme triomphant : "maximum d'efficience, minimum de personnalité". Tel pourrait être le maître mot du Japon de la haute croissance à nos jọrs. Les relations humaines deviennent glaciales et le calcul est une démarche qui semble montrer son adaptabilité au monde moderne.

Les jeunes certes, se méfient des anciennes valeurs et modes de pensée du Japon militariste mais la pression de la société à l'école d'abord, puis sur le lieu de travail ensuite, ne permet pas l'apparition, l'éclosion de valeurs nouvelles originales. Il semble que dans le décor d'un Japon riche, les jeunes puissent s'émanciper et faire ce que bon leur semble. Tout est un leurre car la pression socio-économique qui pèse sur chaque individu ne le permet pas.

Les changements survenus pendant la haute croissance ont été profonds. Cette dernière a eu besoin d'une main-d'oeuvre importante vers la ville. La loi sur l'agriculture la lui a fourni en entraînant non seulement un brusque déclin des populations des campagnes allant vers la ville mais aussi une atrophie et une superficialité des rapports humains qui prévalaient jusqu'alors. L'agriculture a poursuivi sa mécanisation et la télévision a envahi le territoire, le nombre des voitures a augmenté... Tous ces aspects ont exercé une grande influence sur la façon d'éduquer les enfants.

Déjà en 1963, un an avant les jeux olympiques de Tôkyô, un rapport du conseil économique a préconisé au gouvernement de restructurer l'école et la société de façon à repérer, en s'appuyant sur le principe de l'aptitude de chacun, les futures élites dont le pays estimait avoir un impérieux besoin dans la proportion de 3 à $5 \%$. Pour cela, on suggérait des mesures de 
diversification du système éducatif qui aurait privilégié les talents précoces. Cette demande des milieux économiques n'a pas cessé de peser d'années en années sur l'école japonaise.

A cette époque-là, le Ministère de l'éducation a publié un livre blanc sur le thème : "éducation et croissance". Il ne s'agissait pas de la croissance, de l'accroissement de l'esprit humain, de la personnalité de chacun mais plutôt d'une vision où l'homme n'était qu'un terme d'une équation économique plus vaste.

On s'est donc mis à mesurer les performances scolaires de chacun, sur une échelle qui est devenue la référence absolue, le fameux "hensachi", sorte d'échelle de Bint des capacités de chacun permettant une hiérarchisation rapide et claire des individus et de ce fait une orientation professionnelle de ces derniers.

La période de haute croissance économique s'est accompagnée d'une explosion scolaire. Le taux de fréquentation des lycées s'est brusquement élevé.

$\begin{array}{llcc}1960 & 1970 & 1980 & 1985 \\ 57,5 \% & 82,1 \% & 94,2 \% & 94,1 \%\end{array}$

C'est à cette époque que l'obtention du diplôme de fin d'études du second cycle est devenu la condition sine qua non d'un emploi sûr et décent. Inversement, la minorité des $6 \%$ qui n'entre pas au lycée sent peser sur ses épaules une grande suspicion et un handicap professionnel certain.

Pour les $37 \%$ de jeunes qui fréquentent le supérieur, c'est-àdire soit l'université de 4 ou de 2 ans ou les écoles supérieures professionnelles, le problème n'est pas d'exhiber son diplôme 
mais de montrer le lieu où celui-ci a été obtenu. 4 ans d'étude dans une modeste université privée de province où le taux de compétition pour le concours d'entrée est quasiment équivalent au nombre de places disponibles et 4 ans dans l'université de Tôkyô ou de Kyôto ne prédisposent pas les individus à la même carrière.

L'éducation dans ce contexte, est devenu un investissement qui a quelque chose de commercial. Les parents investissent dans l'éducation de leurs enfants. Les marchands d'éducation offrent aux parents de prendre leurs enfants en charge pour la préparation et le "bachotage" des concours.

L'industrie des juku et des yobiko, des écoles parallèles, prospère partout dans le pays et les plus grandes comme Sundaï, Yoyogi Semi ou Kawaï Juku affichent après chaque concours les résultats de leurs élèves reçus dans les bonnes universités. Les patrons de ces établissements, amis du pouvoir, font entendre leurs voix, critiquent fort l'école publique et officielle et se proposent purement et simplement de prendre sa place.

A l'origine, les yobiko étaient des établissements qui s'adressaient aux lycéens qui, ayant raté le concours d'entrée à l'université, n'avaient plus aucune structure pour les accueillir après le lycée. L'image était à l'origine assez négative pour ces établissements dans lesquels se regroupaient les redoublants (ronin).

Pour accéder aux bonnes universités, il est important d'entrer, au préalable, dans les lycées publics ou privés de renom. Mais il en est des lycées comme des universités, on y entre sur concours. Avec le boum sur l'école au moment de la haute croissance, la compétition est devenue acharnée. On a donc ouvert des juku pour aider les collégiens à bien préparer 
les concours d'entrée des grands lycées. Aujourd'hui, il est pratiquement impensable d'entrer dans une université ou un lycée de renom si l'on n'a pas au préalable étudié dans une yobikô ou un juku, et ce, que l'on soit bon élève ou moins bon. Les écoles parallèles sont devenues le détour obligé de la réussite scolaire universitaire et partant professionnelle.

Les concours, contrairement à ce que l'on connaît en France demandent un gros effort de mémoire. Les questions sont nombreuses et il faut choisir les réponses parmi un ensemble de cinq possibles. C'est le système du QCM (question à choix multiples) appelé en japonais le système des ronds et des croix (marubatsu shiki).

Les yobiko et les juku font mémoriser à marche forcée leurs élèves et les divers examens à blanc et galops d'essai sont dûment introduits sur ordinateur et analysés. Les familles des élèves et les intéressés font antichambre et attendent le verdict des ordinateurs omniscients et de leurs pythonisses agréées.

Tout cela bien évidemment est fort coûteux et nombreux sont les parents qui économisent de l'argent dès la naissance des enfants en prévision des frais d'éducation ultérieurs.

Le tableau ci-dessous montre l'augmentation rapide des frais de scolarité dans le second cycle et à l'université, ce qui ne veut pas dire que la période couvrant l'éducation obligatoire n'entraîne aucun frais pour les parents. 
Evolution de la hausse des frais de scolarité (1985-1987) (en dizaine de milliers de yens)

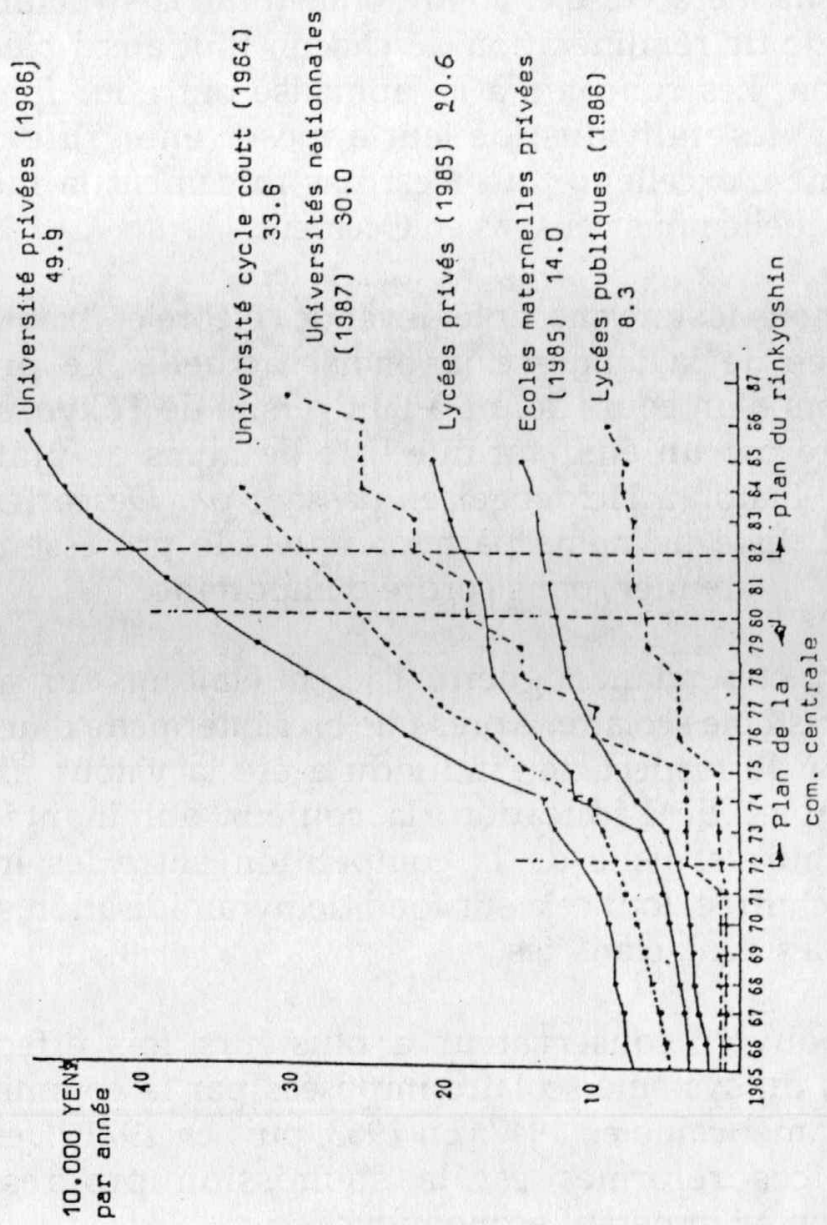




\section{La société du savoir et du diplôme}

Dans le Japon d'aujourd'hui donc, l'aptitude à répondre avec justesse et rapidité à des tests est devenue la mesure entre toutes de la valeur de l'individu qui détermine l'entrée ou non dans une bonne université et partant son destin professionnel. Ce système hiérarchise et conditionne toute la structure sociale et le mode de rémunération de chacun tout aussi bien que sa réputation. Les concours à la japonaise ont pour fonction de "labeliser" les individus, de leur apposer une griffe certifiant une forme d'excellence qui n'est pas forcément la même que celle à laquelle nous pensons en Occident.

Donnons ici une anecdote devenue célèbre et fixant le mode de pensée de la jeunesse japonaise actuelle. Le professeur Keber, lors d'un cours donné à l'université de Tôkyô, s'était vu soumettre par un étudiant une liste de noms de philosophes allant de Platon à Heidegger en passant par Descartes, Hume et Hegel, liste de noms parmi lesquels le professeur devait nommer "le premier" dans l'ordre d'importance.

Avant et pendant la guerre, l'accent était mis sur la loyauté dans le système scolaire. Après une bref intermède dans l'aprèsguerre où le respect de l'individu a été la valeur phare des responsables de l'éducation, la couleur dominante jusqu'à aujourd'hui est celle de la compétition entre les individus dûment chiffrée, tout cela sur fond de pyramidisation sociale et scolaire, les deux étant liés.

Le pouvoir conservateur a plusieurs fois effectué des réformes du système scolaire imposées par la commission de réforme américaine en 1947. En 1963, puis en 1971. L'esprit qui domine ces réformes est la soumission progressive de l'éducation à l'impératif économique. 
La commission de réforme de l'éducation réunie à la demande de M. Nakasone alors premier ministre en 1982, va-telle dans le même sens que les précédentes?

8. La commission provisoire de réforme de l'éducation (Rinkyôshin) et les projets de la classe politique conservatrice liée aux intérêts du monde économique

La haute croissance prend fin avec le choc pétrolier en 1973. Dans les années qui ont suivi, des réformes des finances et de l'administration ont préconisé de pratiquer des coupes franches dans les budgets du bien-être social et de l'éducation pour réduire les dépenses globales de l'état. Peu à peu, on a pris l'habitude de faire supporter aux parents ce qui, pendant la période de haute croisaance, était assumé par l'Etat. Les frais de scolarité ont augmenté dans les universités nationales, la restauration scolaire a été privatisée, la revendication pour diminuer les effectifs des classes au-dessous de 40 élèves a été gelée...

Dans le même temps, le contrôle des manuels scolaires, cette sorte d'imprimatur du ministère de l'éducation, a été renforcé. Dans les manuels d'histoire par exemple le terme jusqu'alors employé "d'invasion" pour caractériser l'acte d'agression japonaise en Chine où l'on a compté plus de 20 millions de morts), a été effacé et remplacé par celui "d'avancée" de l'armée japonaise suscitant l'émotion et les protestations du gouvernement chinois.

Dans l'arrondissement de Nakano à Tôkyô prévalait jusqu'alors l'élection au suffrage populaire des responsables du comité d'éducation dont le rôle est d'administrer la vie scolaire de l'ensemble de l'arrondissement. Le Ministère de l'éducation a déclaré illégal ce mode d'élection et a essayé d'en prendre le contrôle en nommant les responsables de ce comité. 
Des associations influentes de parents d'élèves de la droite japonaise ont demandé que l'on chante à l'école l'hymme national et que l'on hisse le drapeau japonais.

Ces mesures qui concernent plus particulièrement l'éducation sont prises dans un contexte plus large de réformes dont le but est d'alléger le rôle et le fardeau de l'Etat en privatisant (les télégraphes et téléphones, les chemins de fer, la régie des tabacs). Monsieur Nakasone dit vouloir préparer l'an 2000, préparation qui nécessite selon lui de faire le bilan de l'après-guerre (sengo sôkeisan).

L'initiative de ces projets de réforme vient à la fois des milieux financiers et économiques, tout puissants au Japon, et des factions les plus conservatrices du PLD dont M. Nakasone. Un organe consultatif : "entretien sur la culture et l'éducation", présidé par le président de Sony, M. Ibuka, est apparu. Des discussions reposant sur une longue perspective ouverte sur le $X X I^{e}$ siècle ont lieu et incluent économie, société et éducation. Ces échanges d'idées ont pour personnalité centrale souvent des industriels comme celles menées au "club de Kyôto pour penser le monde" (Kyôto zaikai) dont M. Matsushita a été jusqu'à son décès en juin 1989, le personnage central, échanges d'idées qui sont contenues dans les rapports de la "commision d'enquête sur l'économie japonaise", organe de recherche auquel participent les milieux économiques, sont autant d'éléments influents sur l'orientation de la réforme.

Ainsi la commission pour la réforme de l'éducation travaille sur un terrain déjà nivelé. Ses propres membres, mais aussi ceux du club de Kyôto, de "l'Entretien sur la culture et l'éducation" et de la "Commission d'enquête économique" ont un rôle moteur dans ce processus.

Les dirigeants politiques du Japon font un double constat : 
a - la société japonaise est maintenant en tête des grands pays industrialisés pour la production en masse des objets à haute valeur ajoutée.

b- la société japonaise vieillit.

Pour rester en tête, il faut informatiser à outrance la société et l'internationaliser. C'est pourquoi il est nécessaire de changer de système éducatif. La société japonaise du XXI ${ }^{\mathrm{e}}$ siècle doit s'organiser autour d'une élite dirigeante qui, par ses décisions, saura maintenir l'avance du pays. C'est la raison pour laquelle le projet de réforme de la commission provisoire pour l'éducation insiste tant sur la formation des élites. L'un des aspects de l'éducation japonaise, bien connu à l'étranger, est le nombre de tests et de concours que les candidats à l'entrée dans une bonne université doivent subir. Pour les élites, que l'on aura repérées très tôt, il ne sera pas nécessaire de se soumettre à ce bachotage permanent. Pour eux, le gouvernement envisage de créer, un tronc unique allant de la première année de collège à la dernière année de lycée.

Cette "production" des leaders s'appuie sur une éducation morale dans laquelle on insiste sur les aspects immuables de la culture nipponne, sur l'importance de la tradition. A la fois on s'ouvre sur le ${X X X I^{e}}^{e}$ siècle et en même temps on emporte dans le futur ce qui est jugé comme les valeurs fondamentales et inaltérables du Japon comme l'amour de la patrie. Cette conscience alliée à la domination des technologies les plus modernes doit permettre au reste du monde de comprendre la supériorité du Japon. L'un des aspects du nationalisme nippon se retrouve dans le discours de $M$. Nakasone qui attribue à la présence des noirs aux Etats-Unis, le faible coefficient intellectuel général des Américains face au Japon, défini comme un empire homogène. 
Dans cet esprit général, qu'en est-il des problèmes réels et concrets de l'éducation japonaise ? La délinquance, les brimades, le refus d'aller à l'école, les exemples des nombreux laissés pour-compte, les punitions corporelles ? Une telle attitude ne répond en rien aux vrais problèmes de l'école, à la détresse des enfants. Bien au contraire, on insiste sur le fait que les résultats scolaires sont supérieurs au Japon à ce qu'ils sont ailleurs.

Dans son choix évident de donner la parole aux milieux économiques et financiers d'une part, aux écoles parallèles d'autre part et de permettre une critique dure du monde enseignant public, le Jimintô a montré sa double intention de réduire cette poche de résistance que constituent les syndicats de gauche et les enseignants qui y sont affiliés et son budget éducatif puisque l'on donne plus de libertés, de latitude au privé pour se développer. Mais l'enseignement privé et les écoles parallèles ont des frais d'écolage très élevés par rapport au public. Qui va payer ? On peut douter que le gouvernement conservateur va mettre en place un vaste système de bourses pour alléger le budget relatif à l'éducation des familles les moins aisées. Ce seront les parents qui en fin de compte risquent de voir s'alourdir très sensiblement leurs factures "formation et éducation". La dérégulation, la "libéralisation", vent soufflant depuis les Etats-Unis et l'école de Friedman a encore de beaux jours devant elle.

En mettant en place ou en écoutant avec grande attention les multiples comités de réforme et groupes de réflexion dans lesquels le secteur privé est ominiprésent, le gouvernement conservateur montre par là le peu de cas qu'il fait du rôle du parlement. 


\section{L'idéologie de la libéralisation et l'éducation du XXI' siècle}

L'école actuelle n'est pas assez efficace, son taux de rendement est trop faible. En un mot, elle n'est pas assez rentable. Dans une période de faible croissance (entre 3 et $5 \%$ ), l'état manifeste son intention très claire d'alléger son fardeau budgétaire en confiant une partie de l'éducation au secteur privé. Sous le prétexte de mettre l'éducation à l'heure de l'efficacité et du dynamisme, on va assister de facto à la privatisation d'une partie du système éducatif.

Pour les entreprises et le secteur privé, l'éducation est un marché et une marchandise avec laquelle on peut et doit faire du profit. A quand l'école Mitsubishi, Mitsui, ou Toyota, du jardin d'enfant à l'université ? Le marché de l'éducation est d'autant plus prometteur que chacun sait bien qu'au Japon les parents feraient n'importe quoi pour donner à leurs enfants les meilleures chances dans la vie par une formation de qualité. les jukus et les yobikos qui exibent chaque année les résultats de leurs "reçus" poussent de toutes leurs forces pour se faire reconnaitre le droit de dispenser des diplômes scolaires officiels, ce qui reviendrait à les faire reconnaître et les mettre sur le même plan que n'importe quel collège et lycée du pays. L'école parallèle deviendrait école principale.

La philosophie officielle au Japon consiste à lier, à marier au maximum le nationalisme de l'état et celui des entreprises.

Un renversement des valeurs s'est produit qui fait que les entreprises ne considèrent plus l'administration de l'Etat comme ce qui est "en haut", mais comme un ensemble de structures surannées qui freinent leur activité.

Les entreprises japonaises pensent qu'elles sont les piliers qui soutiennent l'Etat et les bureaucrates de tous horizons, dont 
ceux du ministère de l'éducation, mettent trop d'entraves à leur esprit d'initiative et leurs activités. Il est difficile de penser que la marchandisation et la commercialisation de l'enseignement vont produire des écoles originales et des esprits créateurs et indépendants. De plus, ce produit sera payé par les citoyens au prix le plus fort mais tous ne pourront sans doute pas payer.

10. Quelle est la vision de l'homme que défendent les tenants de la libéralisation?

On reproche à l'éducation d'après-guerre d'être trop égalitariste. Il faut, dit-on, introduire la concurrence sur la base des aptitudes réelles parmi les élèves et les enseignants de la même façon que celle-ci existe dans les entreprises. On a dit, de plus, que cette mesure de la valeur et des compétences reposait sur une échelle unique qui ne prend pas en compte la diversité des personnes et des talents et du devenir des individus, de leurs progrès. L'un des grands champions de cette idéologie est $M$. Koyama Kenichi dont on ne peut manquer de citer quelques phrases tirées de ses ouvrages qui permettent de mettre en évidence l'esprit qui l'anime : "L'idéologie Nikyôsô (principal syndicat enseignant) qui rabâche stupidement l'idée d'un enseignement sans laissés pour-compte met en péril la salle de classe..." (Des leçons de la maladie anglaise. PHP 1978). Pour Koyama la véritable tâche est de reconstituer au Japon un enseignement d'élite. Il n'hésite pas à parler d'une vision de l'homme eugéniste lorsqu'il écrit : "Dans la civilisation industrielle d'aujourd'hui, ceux qui ont un quotient intellectuel inférieur à 110 ne sont pas utiles".

Plus loin : "Il faut trouver un moyen qui reconnaisse le droit à ceux qui ont une hérédité supérieure d'avoir plus de trois enfants, les encourager et les aider, et qui force les autres à n'en avoir que deux". Introduction à futurologie , 1967. Dans un tel discours digne de la pensée très conservatrice d'un Platon dans 
la République, Koyama et ses amis osent après parler d'éducation épanouissante et dire qu'elle respecte la personnalité de chacun et que chacun a sa chance. Que deviennent les enfants dont l'épanouissement est tardif ? Que deviennent les handicapés, ceux dont le développement ne va pas au même rythme que les normes que ces pseudo-pédagogues ont établies. (Cf. Que signifie la réforme de l'éducation. Ota et Horio, T, 1985).

La pensée de $M$. Koyama est nourrie par celle de l'économiste américain Friedman qui prône une ingérence minimale de l'état dans le privé. L'Etat doit défendre le privé et la menace essentielle vient des pays socialistes. C'est pourquoi l'Etat minimal qui se désengage au niveau de l'éducation doit avoir un budget militaire fort pour défendre son idéal de société libre. La traduction d'un ouvrage de Friedman Tyrannie $d u$ statu quo a été assurée par $M$. Kato Hiroshi, membre important du club de Kyoto et dans l'introduction japonaise, $M$. Kato dit que M. Nakasone l'a encouragé à tenir compte des idées principales de l'ouvrage pour réfléchir à l'éducation japonaise du XXI siècle. Nous ne sommes pas si loin du slogan nationaliste de Fukuzawa Yukichi, au début de l'ère Meiji au siècle passé, qui voulait "un pays riche, une armée forte" (fukoku kyôhei). Ce nouveau slogan pourrait être "un état qui protège les entreprises, une armée forte, une école privée et élitaire". L'opposition du Ministère de l'éducation est toute provisoire et il arrivera un moment où ce dernier se mettra au service des entreprises comme simple exécutant de leurs choix éducatifs.

\section{Conclusion : les Japonais partagés entre deux projets éducatifs}

L'éducation que proposent de nombreux pédagogues, chercheurs et professeurs en sciences de l'éducation au Japon 
est différente de l'éducation pronée par Messieurs Koyama ou Kato.

Pour les membres de l'Association de l'Education Japonaise de Recherche sur l'Education "Nihon Kyoiku Gakkai" fondée en 1941et qui réunit 3.000 membres en majorité des professeurs d'université ainsi pour les membres de l'Association de Recherche Scientifique sur l'Education "Kyokaken" fondée en 1937 et qui réunit un millier de membres dont de nombreux enseignants du secondaire et du primaire, la conception de l'éducation ne repose pas sur l'économie et les visées nationalistes à peine déguisées, propositions qui sont nées outre Pacifique et donc de mieux avancer masqués.

La conception de l'Association de l'Education Japonaise repose sur les droits de l'homme et les droits de l'enfant. Dans une telle conception l'individu est central et ne peut être sacrifié à aucune stratégie économique même ou surtout si elle est doublée de nationalisme.

La vérité est l'affaire de tous et le savoir et la culture doivent être ouverts sur l'universel. Un enfant n'est pas une chose, un objet dans lequel les parents investissent comme dans une marchandise dans un but égoïste et dans une pure logique de profit. C'est un être à qui il faut enseigner la liberté et l'indépendance d'esprit et non pas en faire, par le biais de l'éducation, un maillon d'une logique marchande nationaliste. Dans l'article 10 de la Déclaration du droit des enfants, il est expressément demandé d'enseigner aux enfants que la culture ou le savoir ne leur appartient pas comme un bien privatif dont ils jouiraient personnellement eux ou leur pays mais que ceux-ci sont des biens communs à tous les hommes et qu'ils doivent se comporter en conséquence. 
Aux milieux patronaux qui demandent à cor et à cri la privatisation de l'enseignement, il faut s'opposer en mettant l'accent sur le fait que l'éducation est une affaire privée qui relève d'une triple responsabilité : celle des enfants, des parents et des enseignants. L'état, loin de contredire cette responsabilité, devrait la favoriser, l'aider au mieux. Un esprit de liberté devrait, au Japon, transformer complètement le système public de l'éducation.

Dire que l'éducation relève d'une affaire privée, c'est reconnaître et permettre aux individus japonais d'exercer le droit de s'occuper de ce qui les regarde au plus haut point. Depuis le début de l'ère Meiji, il y a deux conceptions qui ont prévalu dans le mouvement dit des Lumières japonais (keimô jidai). L'une veut que les réformes et les décisions soient le fait de l'Etat omniprésent et omnipuissant. Dans la conception voltairienne "tout pour le peuple, rien par le peuple", on peut au moins reconnaître, chez les dirigeants, le souci du peuple qui se définit comme total. Dans la première conception de Meiji, la préoccupation première et dernière est l'état et sa toutepuissance. La personne, en tant qu'être autonome producteur d'idées, d'initiatives de valeurs n'existe guère dans une telle conception. Force est de reconnaître qu'un tel esprit perdure aujourd'hui malgré les vicissitudes de l'histoire comme une hydre toujours vivante. Cette conception étatique peut être désignée par le terme "les lumières par en haut".

La seconde conception, accorde toute son importance à la volonté et à l'initiative des citoyens qui correspond à un esprit opposé aux "lumières par en-haut" et que l'on nomme donc les "lumières par en bas". Dans cette filiation, on peut reconnaître les divers mouvements de la liberté et des droits du peuple (Jiyuminken undô) qui sont le véritable ferment de la démocratie japonaise. 
Il faut construire une éducation au Japon qui considère les droits de l'homme et de l'enfant comme la base de l'action. L'éducation doit permettre à chaque être dans quelques pays qu'il soit et à n'importe quel âge de se développer, d'apprendre. Apprendre, s'intruire, se cultiver sont des droits fondamentaux de l'homme. Au lieu de cela, le Japon fait de l'éducation un appareil à classer les individus, selon des critères somme toute pauvres (hensachi) et profondément inégalitaires. Il ne peut y avoir d'éducation sans esprit de justice et de paix et sans souci de vérité et d'égalité. Le véritable progrès est là et non pas dans la mise en place dans une guerre économique qui peut à terme, hélas, engendrer une véritable guerre.

Les nouvelles directives scolaires (gakushû shidô) énoncées par le Ministère de l'Education le 10 février et parues dans la presse le 11 février 1989 justifient ces inquiétudes. Le Ministère donc a décidé de modifier les programmes scolaires, du primaire au second cycle inclus, dans le sens d'un renforcement de l'identité japonaise, du respect du drapeau, de l'hymme impérial devenu national. A partir de 1992, les enseignants devront, pour les cérémonies d'entrée et de sortie des établissements scolaires, chanter l'hymne japonais (kimigayo) debout face au drapeau (Kokki) dans une attitude respectueuse. Un grand nombre d'enseignants et de démocrates attachés à la constitution d'après-guerre craignent une sorte d'escalade dans le retour de ce qu'ils nomment les vieux démons et la tentation sans cesse renouvelée d'un nationalisme qui peut prendre bien des visages au Japon. L'esprit de la réforme de l'éducation entreprise par Nakasone Yasuhiro en 1984 est de faire prendre conscience aux Japonais de leur identité et de leur culture afin de mieux se situer et résister à l'environnement international.

Tout cela bien sûr est habillé dans le discours d'une éducation qui chercherait par-dessus tout la paix entre les pays, la compréhension mutuelle. Il est intéressant de noter que Jean- 
Pierre Chevènement lorsqu'il fut ministre de l'éducation nationale en France a adopté une démarche qui n'est pas très éloignée de celle prônée par Monsieur Nakasone : importance de l'éducation civique, apprentissage de la Marseillaise à l'école...

Dans le même temps, la publication de son ouvrage Apprendre pour entreprendre donne à penser que pour résister aux défis économiques internationaux, il faut que les individus aient une éducation à la fois d'un haut niveau mais également qu'ils aient bien conscience d'appartenir à un champ culturel et historique particulier. C'est une autre manière de décliner le nationalisme. L'économie serait-elle donc la poursuite de la guerre par d'autres moyens? On ne peut manquer d'être inquiet quand on voit le désir des politiciens de réformer l'éducation d'après-guerre sous le prétexte d'une meilleure adaptation aux enjeux actuels et futurs et de fabriquer des hommes nouveaux pour le $X X I^{e}$ siècle. N'y a-t-il pas là un risque de grande tension? Aimer son pays et sa culture, pourquoi pas ? Mais faut-il pour cela l'aimer contre d'autres pays ? Avec le XXI siècle et les diverses réformes de l'éducation ne voit-on pas revenir les nationalismes sous le déguisement de termes habiles comme celui de "l'internationalisation" ? Pour l'écrivain Yamanaka Hisashi les récentes dispositions en matière de programme scolaire ne sont rien d'autres que la réactivation de la morale qui avait cours avant et pendant la guerre. D'un côté on produit des leaders ouverts et créateurs et de l'autre des Japonais obéissants, dociles vis-à-vis des diverses formes d'autorité : les parents, les aînés, les professeurs, l'empereur...

Les laissés pour compte que le système éducatif japonais produit de plus en plus par l'échec scolaire, même si les résultats semblent positifs vus de l'étranger, les phénomènes de brimades, la violence à l'école exercée par les professeurs sur les élèves, par les élèves à l'encontre des professeurs, par les 
élèves entre eux (phénomène du souffre douleur), l'éducation de plus en plus coûteuse, conçue comme une marchandise, dont les couches les plus pauvres n'auront que des succédanés, le refus d'aller à l'école pour des milliers d'enfants chaque année (tôkkô kyôhi) sont les plaies d'un système éducatif dans lequel le respect et l'amour d'autrui en tant qu'individus sont niés.

Dans les projets des cercles et des commissions qui gravitent autour du pouvoir conservateur et des milieux d'affaires on parle peu du bonheur des individus. Le bonheur est aussi un bien auquel les Japonais entendent bien avoir accès. Le bonheur est une idée neuve au Japon. 


\section{SUMMARY}

As in Europe, Japan was influenced after the war by the idea of equality in education. But, this ideal differs from the one prevalent before the war since it focuses on the moulding of free citizens, critical in the respect to matters pertaining to the pre-war state.

However, there is an increasingly more evident awareness of a contradiction between the ideal of equality and the economic utilitarianism in the climate of current economism. This tendency is parallelled by a tendency once again to offer the traditional pre-war values as an option.

The Japanese educational system has become oppressive for children, who are fed massive doses of certain of the principles of competition. Since the Japanese accord a great deal of importance to education, there are parents who are driven to some considerable financial outlay not always within the reach of their fellow-citizens. This is gradually emphasising social differences. This tendency is reinforced by the spread of private education; this is in the process of replacing state schooling, and makes no attempt to instil the universalist values which any teaching worthy of the name must include. Thus, the Japanese are basically divided between two divergent educational blueprints. 